\title{
An efficient iterative pose estimation algorithm
}

\author{
S.H. Or * \\ shor@cse.cuhk.edu.hk
}

\author{
W.S. Luk ${ }^{\dagger}$ \\ Wai-Shing.Luk@cs.kuleuven.ac.be \\ I. King § \\ king@cse. cuhk. edu.hk
}

\author{
K.H. Wong $\ddagger$ \\ khwong@cse.cuhk.edu.hk
}

\begin{abstract}
A novel model-based pose estimation algorithm is presented which estimates the motion of a three-dimensional object from an image sequence. The nonlinear estimation process within iteration is divided into two linear estimation stages, namely the depth approximation and the pose calculation. In the depth approximation stage, the depths of the feature points in three-dimensional space are estimated. In the pose calculation stage, the rotation and translation parameters between the estimated feature points and the model point set are calculated by a fast singular value decomposition method. The whole process is executed recursively until the result is stable. Since both stages can be solved efficiently, the computational cost is low. As a result, the algorithm is well-suited for real time computer vision applications. We demonstrate the capability of this algorithm by applying it to a real time head tracking problem. The results are satisfactory.

keyword : Pose Estimation, singular value decomposition, real time vision and Human-computer Interface.
\end{abstract}

\footnotetext{
*Computer Science and Engineering Department, The Chinese University of Hong Kong, Shatin, N.T.,Hong Kong. Fax: 8522603 5024, Tel. No.:852 26098402 .

${ }^{\dagger}$ Katholieke Universiteit Leuven, Departement Computerwetenschappen, Celestijnenlaan 200A, B-3001 Heverlee,

${ }^{\ddagger}$ same as *

$\S_{\text {same as * }}$
} Belgium. 


\section{Introduction}

Tracking the pose (position and orientation) of a moving object from an image sequence is useful in applications such as photogrammetry, passive navigation, industry inspection and human-computer interfaces $[11,2]$. However, this is a difficult problem because of the following reasons. Firstly, the depth information has been transformed by a nonlinear mapping to yield the foreshortening effect in the captured image. Moreover, the rotation of the object in 3D space leads to a nonlinear formulation which increases dramatically the computational requirement for generating the solution.

In this paper we are interested in the model based pose estimation problem or the $2 \mathrm{D}$ to $3 \mathrm{D}$ problem as categorized by Huang [12]. That is, when the 3D structure of the object being investigated is known and its 2D projected image is available, we want to compute the pose of the object. In practice, the model or the 3D positions of the feature points of the object can be measured manually before tracking begins. A $2 \mathrm{D}$ correlation method can be used to track the movements of the projected 2D feature points. Using these information we would like to find the rotation $(R)$ and translation $(T)$ of the object in 3D. A number of techniques have been developed by previous researchers $[7,23,18,14,13,6,2,8]$ to solve this problem. One way is to compute the motion information directly from the optical flow [3, 13], which require massive computation power. In [7], a closed form solution for the pose from six points is derived. The method is extended to the estimation of more points by a consensus of sampling from the set. Robustness is achieved in this method at the expense of intensive computation of motion information from sampled subsets of the points.

Another method to increase the robustness is the use of the method of least squares. Depending on the formulation (using Euler angles or quaternions to represent rotation), various methods can be used to recover the pose from exact data. However, the problem will become a nonlinear least squares one when noise exists. Iterative algorithms such as the Newton-Raphson method [14] are applied to find the solution but with the drawback of being heavily dependent on the initial guess.

It is observed that a long image sequence can provide more information for the estimation process to suppress noise. Extended Kalman filter is used to model the problem as a recursive estimation process by Broida et al. [5, 4] and Azarbayejani et al. [2]. The authors demonstrated that the pose of a moving object can be successfully recovered. However, the Kalman Filter method is found to be successful in those applications where the observed objects would not change their appearance rapidly, 
e.g. aerospace and vehicles applications [14]. In other applications such as human motion tracking, owing to the complexities of the motion involved, more work is needed to increase the robustness of the overall tracking system.

Our interest is in developing an efficient algorithm which is suitable for various applications on human motion analysis. This area is characterized by the rapid motion of the object concerned and high probability of occlusion/re-appearance of part of the object in the subsequent frames.

Specifically we are interested in solving the 2D to $3 \mathrm{D}$ problem as mentioned by Huang[12]. We notice that there is already a solution for the $3 \mathrm{D}$ to $3 \mathrm{D}$ problem as mentioned in [1]. That is when the 3D structure of an object is known and the 3D locations of the features in the new positions are available, we can use the method of singular value decomposition (SVD) to find out the motion parameters $[1,19,10]$. A least squares solution is found. However the algorithm will sometimes give a wrong (reflection of the correct answer) solution when the input data are severely corrupted by noise. This was fixed by Umeyama [19] and later shown to be valid even when both point sets are noisy [10]. In this paper, we propose an algorithm which extends the above methods to handle the problem of recovery of motion information from $2 \mathrm{D}$ projections of a given 3D point set. Our solution consists of the following two modules. (1) From the 2D features on the image plane, we estimate the locations of the features in 3D. (2) Using the estimated 3D information we use the method of SVD as in [1, 19, 10] to develop the motion parameters of the object. The above two processes are executed recursively until the solution is stable. Our contribution here is that our algorithm requires significantly reduced computation compared with other techniques [14,2] under the situation of large number of feature points, say over 20 .

The rest of this paper is organized as follows. In Section 2, the model-based pose estimation problem is formulated. Section 3 contains the first version of our algorithm together with the convergence analysis of the algorithm. The weakness of that algorithm is identified and a more robust algorithm is then proposed. In Section 4, the performance of the improved algorithm is compared with two established approaches, namely the Gauss-Newton method and the Extended Kalman Filter method. In Section 5, we tested our algorithms on both synthetic and realistic image data. A head tracking application is implemented to test the validity of our approach on realistic images. Finally some possible enhancements of our algorithms are discussed in Section 6. 


\section{Problem formulation}

Consider a camera with the origin of the focal plane placed at $(0,0, f)$, as shown in Fig. 1 , where $f$ is the focal length of the plane. Assume that a set of $N$ model points $\left\{P_{i}, i \in 1,2, \ldots, N\right\}$ in 3D space are given, which represents an object model. A rigid transformation (a rotation followed by a translation) is applied to this point set, yielding $\left\{P_{i}^{\prime}\right\}$, which is then projected on the image plane, giving $\left\{\left(X_{\mathbf{Q}_{i}}, Y_{\mathbf{Q}_{i}}\right), i \in 1,2, \ldots, N\right\} .{ }^{1}$ These $2 \mathrm{D}$ feature points in our $3 \mathrm{D}$ coordinate system are given by

$$
Q_{i}=\left(X_{\mathbf{Q}_{i}}, Y_{\mathbf{Q}_{i}}, f\right)
$$

Let the $3 \mathrm{D}$ coordinates of the transformed points $P_{i}^{\prime}$ be $\left(X_{P_{i}^{\prime}}, Y_{P_{i}^{\prime}}, Z_{P_{i}^{\prime}}\right)$. The coordinates of $P_{i}^{\prime}$ and $\mathbf{Q}_{i}$ are related by

$$
X_{\mathbf{Q}_{i}}=f \cdot \frac{X_{P_{i}^{\prime}}}{Z_{P_{i}^{\prime}}}, \quad Y_{\mathbf{Q}_{i}}=f \cdot \frac{Y_{P_{i}^{\prime}}}{Z_{P_{i}^{\prime}}} .
$$

In this arrangement, any point $\mathbf{Q}_{i}$ tracked in the image will give an inverse projection ray from the focal centre with unit vector $\nu_{i}$ given by $\left(X_{\mathbf{Q}_{i}}^{2}+Y_{\mathbf{Q}_{i}}^{2}+f^{2}\right)^{-\frac{1}{2}}\left(X_{\mathbf{Q}_{i}}, Y_{\mathbf{Q}_{i}}, f\right)$. The actual coordinates of this point in $3 \mathrm{D}$ space are given by

$$
P_{i}^{\prime}=d_{i} \nu_{i}
$$

where $d_{i}$ is the depth (a scalar) of the actual object from the perspectivity center.

The problem of pose estimation can be described as follows. Given $\left\{\mathbf{Q}_{i}, i \in 1,2, \ldots, N\right\}$, and a 3D model $\left\{P_{i}, i \in 1,2, \ldots, N\right\}$ where $P_{i}=\left(X_{P_{i}}, Y_{P_{i}}, Z_{P_{i}}\right)$ are the coordinates of the model points at a reference instant. We seek $R, T$ and $\left\{d_{i}\right\}$ such that the following measurement function

$$
e^{2}\left(R, T,\left\{d_{i}\right\}\right)=\sum_{i=1}^{N}\left\|d_{i} \nu_{i}-\left(R P_{i}+T\right)\right\|^{2}
$$

is minimized. The scalar $d_{i}$ corresponds to the depth $(\mathrm{Z})$ which determines where in $3 \mathrm{D}$ space should the actual point be located along the projection ray.

In the formulation above, least squares minimization methods can be applied to the $N+6$ parameter space to find the minimum of the measurement function $e^{2}$ where $N$ is the number of feature points of the object. This approach is adopted by [17,21]. Since the original 3D coordinates are being transformed by a perspective projection, the objective function is thus nonlinear. To solve for the

\footnotetext{
${ }^{1}$ In this paper, we use bolded font to represent the $2 \mathrm{D}$ vector on the image plane.
} 
solution, nonlinear minimization methods are required, which are usually time consuming as the number of points increases.

\section{Our algorithm}

Recently some work has been presented which focus on using the projection rays as a guide to determine the pose of an object in an image [22]. However, that approach used the iterative point matching method, which demands much computational effort. Inspired by the inverse projection ray approach, we propose that the minimization process can be broken down into two stages: the first stage will estimate the position of all the feature points in the $3 \mathrm{D}$ space, i.e. $\left\{d_{i}\right\}$. The estimated point set will be passed to the second stage, which is a least squares fitting of the $3 \mathrm{D}$ model set and estimated 3D point set. The above procedure is repeated until the result converges. By dividing the estimation process into two stages, the size of the solution space in each stage is much reduced and the cost in locating the solution is minimized significantly. The resulting algorithm is very efficient and can run in real time.

\subsection{Depth approximation stage}

We would like to estimate the approximate depth values $d_{i}$ for all the feature points in this stage. Consider Fig. 1, which shows a transformed point $P^{\prime}$ and its original position $P$. The point $P^{\prime}$ falls on the projection ray which emanates from the origin through the image point. It is not possible to have an accurate prediction of where on the projection ray the point would reside. However, the point must be on the projection ray, and by the assumption that the displacement should be small, we take the perpendicular intersection of the original point with the projection ray as the estimated position in the first version of our algorithm. From the figure, assuming that the unit vector along the projection ray is $\nu$, the perpendicular projection is given by

$$
Q^{\prime}=\left(\nu^{\mathrm{t}} P\right) \nu
$$

In the above formula, the superscript "t" denotes the transpose of a vector. As seen in Fig. 1, the estimated position of the feature point in the 3D space is only the perpendicular intersection of the model point with the projection ray, therefore, it may not correspond to the actual location of the 
transformed point. However, after each iteration, the model will be transformed closer to the projection ray. The algorithm will eventually converge to a solution when the sum of the perpendicular distances from the estimated feature points to the projection ray becomes minimal.

\subsection{Least squares fitting}

In this stage, we minimize the following objective function

$$
e^{2}(R, T)=\sum_{i=1}^{N}\left\|Q_{i}^{\prime}-\left(R P_{i}+T\right)\right\|^{2}
$$

where $Q_{i}^{\prime}$ is the perpendicular projection given by eq. (4) obtained in the previous estimation stage and $P_{i}$ is the original (model) point. As mentioned above, various efficient algorithms are available for the fitting of two $3 \mathrm{D}$ point sets. We choose the singular value decomposition method $[1,19]$ due to its robustness in noise handling and that only a $3 \times 3$ matrix decomposition procedure is needed.

The algorithm is described below. The readers are referred to $[19,1]$ for details.

Arun/Umeyama algorithm for 3D to 3D motion estimation:

Input: $\left\{P_{i}, i \in 1,2, \ldots, N\right\}$ and $\left\{P_{i}^{\prime}, i \in 1,2, \ldots, N\right\}$ in 3D space. Output: Rotation matrix $R$, translation vector $\mathbf{T}$ and scalar value $c$ such that

$$
\sum_{i=1}^{N}\left\|P_{i}^{\prime}-\left(c R P_{i}+T\right)\right\|^{2}
$$

is minimized.

1. Compute the following

$$
\begin{aligned}
\mu_{P} & =\frac{1}{N} \sum_{i=1}^{N} P_{i} \\
\mu_{P^{\prime}} & =\frac{1}{N} \sum_{i=1}^{N} P_{i}^{\prime} \\
\sigma_{P}^{2} & =\frac{1}{N} \sum_{i=1}^{N}\left\|P_{i}-\mu_{P}\right\|^{2} \\
\sigma_{P^{\prime}}^{2} & =\frac{1}{N} \sum_{i=1}^{N}\left\|P_{i}^{\prime}-\mu_{P^{\prime}}\right\|^{2} \\
H & =\frac{1}{N} \sum_{i=1}^{N}\left(P_{i}^{\prime}-\mu_{P^{\prime}}\right)\left(P_{i}-\mu_{P}\right)^{\mathrm{t}}
\end{aligned}
$$


2. Find the SVD of $H$ :

$$
H=U W V^{\mathrm{t}}
$$

where $U$ and $V$ are $3 \times 3$ orthonormal matrices, and $W$ is a $3 \times 3$ diagonal matrix whose diagonal elements contain the eigenvalues of $H$.

3. If the rank of $H>2$, the optimal transformation is given by

$$
\begin{gathered}
S=\left\{\begin{array}{rr}
I & \text { if } \operatorname{det}(H) \geq 0 \\
\operatorname{diag}(1,1,-1) & \text { if } \operatorname{det}(H)<0
\end{array}\right. \\
R=U S V^{\mathrm{t}} \\
c=\frac{1}{\sigma_{P}^{2}} \operatorname{tr}(W S) \\
T=\mu_{P^{\prime}}-c R \mu_{P}
\end{gathered}
$$

else if the rank of $H=2$,

$$
\begin{aligned}
S=\left\{\begin{aligned}
I & \text { if } \operatorname{det}(U) \operatorname{det}(V)=1 \\
\operatorname{diag}(1,1,-1) & \text { if } \operatorname{det}(U) \operatorname{det}(V)=-1
\end{aligned}\right. \\
R=U S V^{t} \\
c=\frac{1}{\sigma_{p}^{2}} \operatorname{tr}(W S) \\
T=\mu_{P^{\prime}}-c R \mu_{P}
\end{aligned}
$$

In the above, $\operatorname{det}(x)$ is the determinant of $x, \operatorname{tr}(x)$ is the trace of a matrix $x$.

In Umeyama's formulation, a scaling parameter $c$ is also recovered. In our algorithm, we fix the value of $c$ at one to reflect the fact that the object size does not change in subsequent images.

\subsection{Algorithm I}

The complete algorithm is as follows:

Input: 
$\left\{P_{i}, i \in 1,2, \ldots, N\right\}: 3 \mathrm{D}$ coordinates of the feature points (or the model) of the object, $\left\{\mathbf{Q}_{i}^{\prime}:\left(X_{\mathbf{Q}_{i}^{\prime}}, Y_{\mathbf{Q}_{i}^{\prime}}\right), i \in 1,2, \ldots, N\right\}:$ transformed feature point coordinates on the image plane.

Procedure:

While (change in $R$ or $T$ not less than some threshold values)

1. Estimate $d_{i}$ by $\nu_{i}^{\mathrm{t}} P_{i}$ where $\nu_{i}$ is the unit vector along the projection ray formed by image point $\mathbf{Q}_{i}^{\prime}$ and the origin.

2. Perform a least squares fitting to eq. (5) to estimate $R$ and $T$ by the singular value decomposition method $[1,19]$.

3. Update $P_{i}$ by $P_{i} \leftarrow R P_{i}+T$.

end while

\subsection{Convergence analysis}

We now perform a convergence analysis of our algorithm. The analysis of the convergence condition of a particular algorithm is usually not treated in most pose estimation literature. However, it is an important criterion for the evaluation of algorithms. Many motion estimation algorithms are very sensitive to noise. Moreover nonlinear algorithms usually lead to wrong results if the initial guess is not sufficiently close to the solution. It is therefore of interest to estimate the effect of different motion parameters on the recovered values.

Assuming that a model point $P$ is transformed to another location $P^{\prime}$ by a rigid body transform, the perpendicular projection of $P$ onto $P^{\prime}$ is $Q^{\prime}$ (see Fig. 1), and $P^{\prime}$ is given by

$$
P^{\prime}=R P+T
$$

From Fig. 1, $Q^{\prime}$ can also be written as

$$
Q^{\prime}=P^{\prime}\left(1-\frac{\left(P^{\prime}-P\right)^{\mathrm{t}} P^{\prime}}{\left(P^{\prime}\right)^{\mathrm{t}} P^{\prime}}\right) .
$$

The second term on the right hand side of the above equation, which is the fractional difference between the estimated position and the true one, can be treated as the error in prediction. By studying the dependencies of this term on feature configurations and motion characteristics, the performance of our algorithm can be readily characterized. 
The second term inside the parenthesis on the right of eq. (7) can be written as

$$
\begin{aligned}
\varepsilon & =\frac{\left(P^{\prime}-P\right)^{\mathrm{t}} P^{\prime}}{\left\|P^{\prime}\right\|^{2}} \\
& =\frac{(R P)^{\mathrm{t}} R P+(R P)^{\mathrm{t}} T+T^{\mathrm{t}}(T-P)-P^{\mathrm{t}} R P}{(R P)^{\mathrm{t}} R P+2(R P)^{\mathrm{t}} T+T^{\mathrm{t}} T} .
\end{aligned}
$$

The prediction error term $\varepsilon$ depends on the values of $R, P$, and $T$. From the above equation, we would expect that for a large ratio of $\|T\|$ to $\|P\|$, the algorithm would converge to a wrong result as $\varepsilon$ approaches one.

From the above, we see that our algorithm would converge to wrong results as the ratio $\|T\| /\|P\|$ increases. However, imagine a scene in which an object moving at a fast speed, the feature points taken between successive frames would probably result in significant distances apart. Since our algorithm will only try to bring the model as close to the projection ray as possible, this would result in the model being brought to a local minimum where the algorithm will be stuck. A more robust enhancement is therefore needed to handle the translational movement.

Recall that our original algorithm can be viewed as follows:

Iterate until convergence

1. Construct the initial estimate by projecting the model points individually onto the line formed by the camera origin and feature points,

2. Fit a rotation matrix and a translation vector to account for the transformation between the estimated set and model set.

We can see that the initial guess plays an important role in the overall estimation process. Assuming there is no motion in the model points (i.e. the rotation and translation have already been determined and the model points have been updated), the variables $\left\{d_{i}\right\}$ now remain to be determined. They can be viewed as the parameters obtained from the minimization of $\Theta$, where

$$
\begin{aligned}
\Theta & =\sum_{i=1}^{N}\left\|d_{i} \nu_{i}-P_{i}\right\|^{2} \\
& =\sum_{i=1}^{N}\left(d_{i}^{2}-d_{i} \nu_{i}^{\mathrm{t}} P_{i}-d_{i} P_{i}^{\mathrm{t}} \nu_{i}+P_{i}^{\mathrm{t}} P_{i}\right) .
\end{aligned}
$$


In the above formula, $\nu_{i}$ is the unit vector of the projection ray (with the property $\nu_{i}^{\mathrm{t}} \nu_{i}=1$ ).

Differentiating the above equation with respect to $d_{i}$ and setting the partial differential equals to zero and rearranging terms, we have

$$
d_{i}=\nu_{i}^{\mathrm{t}} P_{i}
$$

which is the same form as a dot product of $\nu_{i}$ with $P_{i}$.

We can see that our original algorithm is working in the following way:

1. Assume the object has no motion, determine $\left\{d_{i}, i \in 1,2, \ldots, N\right\}$.

2. Assume $\left\{d_{i}, i \in 1,2, \ldots, N\right\}$ are determined, estimate $R$ and $T$.

3. Update the state of the solution and iterate again.

\subsection{Algorithm II}

At this stage, we modify the minimization function in eq. (10) so as to increase the robustness of our algorithm. The idea is to add the translation term into the minimization function in stage 1 so that the predicted position $\left\{d_{i}\right\}$ for $i \in 1,2, \ldots, N$ will be more accurate.

The improved algorithm is as follows:

1. Minimize the function below to estimate $\left\{d_{i}, i \in 1,2, \ldots, N\right\}$ :

$$
\sum_{i=1}^{N}\left\|d_{i} \nu_{i}-\left(P_{i}+T\right)\right\|^{2} .
$$

The resulting $\left\{d_{i}, i \in 1,2, \ldots, N\right\}$ and $T$ is given by:

$$
\begin{aligned}
d_{i} & =\nu_{i}^{\mathrm{t}}\left(P_{i}+T\right) \\
T & =-\left(\sum_{i=1}^{N} A_{i}\right)^{-1}\left(\sum_{i=1}^{N} A_{i} P_{i}\right)
\end{aligned}
$$

where $A_{i}$ is a 3 by 3 matrix given by

$$
A_{i}=I-\nu_{i} \nu_{i}^{\mathrm{t}}
$$

For the derivation of the above formula, please refer to Appendix I. 
2. Using the estimated $\left\{d_{i}, i \in 1,2, \ldots, N\right\}$ in the previous stage, apply the SVD method to determine the rigid transformation $R$ and $T$.

3. Update $\left\{P_{i}, i \in 1,2, \ldots, N\right\}$ by $P_{i} \leftarrow R P_{i}+T$.

Note that $A_{i}$ 's are not needed to be stored explicitly. Firstly, each $A_{i}$ depends only on $\nu_{i}$, which is fixed before the execution of our algorithm. Therefore the value of $\left(\sum_{i=1}^{N} A_{i}\right)^{-1}$ in eq. (14) can be precomputed before the iterations, which avoids the expensive inverting operation of a matrix. Moreover, the matrix vector product $A_{i} P_{i}$ can be computed efficiently using the formula $P_{i}-\nu_{i}\left(\nu_{i}^{\mathrm{t}} P_{i}\right)$. As a rough estimate for the computational requirements at each iteration, for $N$ data points, our algorithm needs only about $41 \mathrm{~N}$ arithmetic operations (additions, subtractions and multiplications) and one singular value decomposition operation for a $3 \times 3$ matrix, that is about a hundred floating point operations. We also noticed the algorithm by DeMenthon and Davies [6] which requires only $24 N$ arithmetic operations and two square root operations. However, our argument for the efficiency is still valid by the following reasons. Firstly, in the approach used by DeMenthon and Davies, the algorithm requires an approximate pose estimate from the previous stage, which would inevitably contribute to the computational requirement. Moreover, their method does not guarantee the orthonormality of the resulting rotation matrix. Whereas our algorithm uses the singular value decomposition, which provides an orthonormal result, hence improves the accuracy and thus justifies the increase in computation. Hence we conclude that the resulting algorithm is accurate and efficient.

\section{Performance comparison}

Various approaches have been applied successfully to the problem of motion analysis. In this section, we compare the performance of two established approaches with our algorithm. The main interest here is the computational efficiency since the main concern during the motion tracking application would probably be the timing requirement as well as accuracy and stability. We chose the Gauss-Newton method (by Lowe [14]) and Extended Kalman Filter method (by Broida et al. [4] and Azarbayejani et al. [2]) due to their robustness and computational efficiency. 


\subsection{Gauss-Newton method}

Lowe $[14,15]$ used the Gauss-Newton method augmented with stabilization with prior variance to solve the pose tracking problem. In this method, the following matrix equation is being solved:

$$
\left[\begin{array}{c}
J \\
W
\end{array}\right] h=\left[\begin{array}{c}
-E \\
0
\end{array}\right]
$$

where $h$ is the unknown vector of corrections to be made to current estimate, $E$ is the error between the current measurement and that of model prediction, $J$ is the Jacobian matrix of the error with respect to the parameters and $W$ is a diagonal weighting matrix which stabilizes the solution. It should be noted that Lowe's approach minimizes the errors between the model prediction in 2D image space, while our formulation fits a rigid transformation between the previous model and an estimated one in 3D object space. In our experiment, Lowe's approach is written in $\mathrm{C}$ and is derived from the package on rigidity checking from the kind offer by McReynolds $[16]^{2}$.

\subsection{Extended Kalman Filter method}

The Kalman filter has been investigated by many researchers for solving the motion tracking problem $[2,4]$. It features an optimal least squares solution to the problem along with the prediction mechanism. Our implementation followed that of Azarbayejani et al. [2]. The main advantage of Azarbayejani et al.'s formulation is that it only used one parameter per feature which reduces the size of measurement matrix and thus is computationally more efficient.

The Extended Kalman Filter algorithm was implemented in $\mathrm{C}$ and was tested together with the approach by Lowe as well as ours.

\subsection{Results and discussions}

All the simulations were performed on a SUN Ultra 1/170 workstation. The same data set was used which involved both translation and rotation of two to four planes in $3 \mathrm{D}$ space. The test set was started with 7 points, then 10 points which lie on the same plane. Five points were added after each test and a new plane was added after each 10 points increment. The motion trajectory consists of increasing values in both rotation and translation for 100 frames. Translation in $x$ axis increased from

\footnotetext{
${ }^{2}$ The package can be downloaded from the following site ftp://ftp.cs.ubc.ca/pub/local/danm .
} 
zero to 0.5 in the first 50 frames whereas translation in $y$ axis is in the negative direction and linearly decreased to -2.0 for the whole sequence. Angles with respect to $y$ axis and $z$ axis are slowly increased from zero to around 0.4 in the last 50 frames. Monte Carlo simulation was applied to the data and Gaussian random noise with zero mean and standard deviation of one unit is added to the coordinates of each feature point. The plots of average time per frame used by various approaches are shown in Fig. 2. In the plot, our algorithm is represented using the title "PRA" which stands for "Projection Ray Attraction". The computational advantage of our approach is clear from the figure. It can be seen that the time required by Lowe's approach increases linearly with the number of points whereas our approach remains roughly the same. The main reason is that the Gauss-Newton method requires the solution of an $(N+6) \times(N+6)$ matrix, where $N$ is the number of feature points measured. This matrix inverse operation is computationally the most demanding step. The long computation time of the Extended Kalman Filter stems from the fact that it has to solve for a $2 N \times 2 N$ matrix.

However, it should be noted that despite the computational efficiency as shown above, our current approach still lacks the sophistication in other approaches. For example, the Extended Kalman Filter algorithm has the properties of motion prediction together with the recursive estimation of structure as well as focal length. Another example is the stabilizing properties of Lowe's approach, which is important during long sequence tracking. In view of the generality of our formulation, it is not a difficult problem and we will incorporate the above features in the future.

\section{$5 \quad$ Experiments}

\subsection{Synthetic data}

To test the validity of the above formulae, we use a randomly generated point set of 16 points inside a unit cube. The point set is transformed (rotated and translated) and projected on an image plane of unit focal length. Algorithm II is then applied to recover the transformation parameters. We vary one of the parameters, say roll angle while keeping all the other parameters at zero. The resultant plots for different Euler angles are shown in Fig. 3. We have also tested the performance for different translation values and the results are satisfactory. Since the algorithm can estimate the translation accurately, we did not show the plot here. It is observed that the recovered Euler angle becomes unreliable after one or more of the input angles exceed 20 degrees. Also note that the translation terms can 
be unambiguously recovered even for a large value compared with the magnitude of original model vectors. We have also performed the same set of experiments on algorithm I. The same performance on recovered rotation is observed while the estimated translation quickly drops to zero when the translation increases as shown in Fig. 4. This is to be expected according the analysis in Section 3.4.

The performance of our algorithm under noisy environment was also investigated. Two sets of experiments were performed. We first describe the setup since they are common for both experiments. A number of points are randomly generated in the $3 \mathrm{D}$ space such that they will project on an image plane of size 2 by 2 . These model points are then transformed by a rotation about the axis $(1,1,1)$ by $6^{\circ}$ followed by a translation of $(5.0,3.0,6.0)$. The transformed points are then projected on the image plane. The image coordinates are then digitized to a screen resolution of $512 \times 512$. The process of digitization introduces noise in this case. 100 random scenes are generated according to this setup and the digitized coordinates are used together with the original generated 3D model to determine the pose.

The errors shown in the following are all relative errors. We used quaternions to represent the rotation such that all the estimated results are in vector form. The relative error of a vector is defined by the Euclidean norm of the difference between the estimated and the true vector divided by the Euclidean norm of the true vector. The first set of experiments tests the performance of the algorithm under different number of points in the scene. The results are shown in Fig. 5. From the plot, it is observed that the estimated rotation is relatively stable with respect to the variation of the number of points whereas the translation has the greatest improvement from 8 to 12 points. In addition, the digitization only introduces a small effect on the estimated results, as can be seen in the range of errors it is only within 3 percents.

Another set of experiments simulates the measurement noise by introducing an offset value to both digitized $x$ and $y$ coordinates. These offset values are normal distributed with a variance of $k$ pixels where $k$ is the parameter to be varied in the experiments. 16 points are used in this set of experiments. Fig. 6 shows the results and our algorithm again is quite stable under the situation of increasing noise.

\subsection{Real image testing}

Pose tracking involves continuous monitoring of the pose of an object from the input image sequence. Our algorithm can easily be adapted for continuous tracking by performing estimation between suc- 
cessive image frames. We tested our algorithm by applying it to track the head of a person in an image sequence. Our implementation is done on an SGI Indy workstation. To verify the correctness of the recovered pose information, we reproduce the same motion on the workstation at the same time. Though it is difficult in this case to estimate the performance figure of the algorithm, an overall estimate of the usefulness of the algorithm can be obtained.

\subsubsection{Calibration}

A calibrated data point set for the face to be tracked is needed in our experiment. Two methods can be used. In the first approach, one can measure directly the $3 \mathrm{D}$ coordinates of the feature points on the face with respect to a reference point, say the nose tip. The drawback of this approach is that careful measurements are needed and that the process is intrusive. The second approach uses the structure from motion algorithm. We used the second approach, following the algorithm by Szeliski [17]. An image sequence of the object is obtained and the feature points of the model are selected and tracked throughout the sequence to generate a batch of $2 \mathrm{D}$ image coordinates of these feature points. The resulting data is input to the batch processing algorithm which performs a non-linear minimization (Levenberg-Marquardt method). The algorithm will estimate the focal length $f$, the model point set $\left\{P_{i}, i \in 1,2, \ldots, N\right\}$ as well as the motion parameters. This process usually requires much more time. For the case of a total of 250 data points for a synthetic head model, the total time needed is over 10 hours on a SUN-Sparc10 workstation. In our real image experiment, only twelve points are used. All points are selected on the criteria that they can be tracked unambiguously throughout the sequence so as to increase the accuracy of the recovered pose. We use four points for the head boundary including ears, four points for the mouth, and four points for each of the eyebrows.

\subsubsection{Initialization}

The user first clicks on the captured image with a mouse to select the feature points. The selected feature points must be the same as those taken in the calibration stage. These selected feature points are then tracked by the normalized correlation method to give coordinates in successive frames. The correlation window is of size $5 \times 5$ whereas the search window is of size $7 \times 7$. The point inside the search window with the highest correlation score is chosen as the feature point location in the subsequent frame. For the first frame, the captured point sets are used to estimate the proper pose adjustment of 
our model set. The pose adjustment is necessary since the pose of the model point set obtained in the calibration stage is not at the same pose with that in the captured image now. This adjustment will bring the model point set to the most likely pose with that in the captured image now. It is inevitable that error in selecting feature exists due to disagreement in the location of the feature selected and that in the calibration stage. However, according to our experience, this discrepancy is minor in that our algorithm can still maintain the tracking over a long period, say over 100 frames. Once the pose adjustment is completed, the pose tracking procedure can be applied to the human object.

\subsubsection{Tracking}

Once the pose adjustment is completed, our algorithm will generate the pose estimates in all subsequent frames. The pose estimates are in the form of a rotation matrix and a translation vector. We transform the rotation matrix into the Euler angle format. These information will be used to control a synthetic head model $^{3}$. Results are shown in Fig. 7. Fig. 7(a) shows a snapshot during initialization. The user is sitting right in front of the camera and the feature points on the face are being selected. Once the initialization is done, the user is allowed to move freely within the view of the camera. Fig. 7(b) shows another snapshot in which the user is rotating his head to the right and tilting upward. It can be seen that the synthetic face can produce visually the same pose at the same time. Currently our system can achieve a processing speed of around 12 frames per second for a 320 by 240 image size. The performance figures are obtained under the condition of the tasks of capturing, feature tracking, motion estimation and controlling of the synthetic character all being done on the same SGI Indy workstation.

\section{Discussion}

In the experiments on head tracking, we found that our algorithm can effectively recover the motion information from the images. The main advantage of our algorithm is that it is computationally efficient. In addition, by performing the fitting of the model set in $3 \mathrm{D}$ object space and not on the image plane, a larger range of convergence is achieved which enables our algorithm to be more stable with respect to different initial guesses. However, our algorithm also has the following drawbacks.

\footnotetext{
${ }^{3}$ The synthetic character is a public domain implementation of the facial animation work by Keith Waters [20].
} 
Firstly, due to the fact that our algorithm is formulated under the situation of two frames only, thus the error in the estimation will be accumulated in the subsequent frames and may lead to failure after a large number of frames. Moreover, in the current formulation, the problem of occlusion is not being handled. Finally it is found that the recovered pose of the head will slowly lose track when one or more of the correlated point fails to give the correct movement.

For the first problem, one solution is to bootstrap the algorithm itself after a number of frames. This is possible since our algorithm has quite a large range of convergence (approximately 40 degrees for the rotation angles as seen in Section 5.1). Another solution is to cross-check the pose estimation result with two or more frames earlier so as to minimize the error itself. The second problem demands a further enhancement of our algorithm to handle the occlusion problem, which is not too difficult due to the model based nature of our algorithm [9]. We are currently working in this direction. When the above problems are solved, we can produce a robust head tracking system. An exciting application of head tracking is in low bit rate video signal compression.

\section{Conclusion}

An efficient pose estimation algorithm has been presented. By breaking down the pose estimation process into two separate linear stages, the computation cost is significantly reduced. A real time pose tracking system has been produced. We also analysed the input tolerance of our system and found that the range of input variation in the rotation angles should not exceed the range of 40 degrees approximately for each angle. Tests on synthetic data as well as real world head tracking have demonstrated the effectiveness of our algorithm.

\section{Appendix I: Derivation of Initial Guess for Improved Algorithm}

Given $\left\{P_{i}\right\}$ and $\left\{\nu_{i}\right\}$, we want to minimize the function below to establish the initial guess $\left\{d_{i}\right\}$ :

$$
\begin{aligned}
\Theta & =\sum_{i=1}^{N}\left\|d_{i} \nu_{i}-\left(P_{i}+T\right)\right\|^{2} \\
& =\sum_{i=1}^{N}\left[d_{i} \nu_{i}-\left(P_{i}+T\right)\right]^{\mathrm{t}}\left[d_{i} \nu_{i}-\left(P_{i}+T\right)\right]
\end{aligned}
$$


Applying partial differentiation to $\Theta$ with respect to $d_{i}$ and setting it to zero, we have

$$
\frac{\partial \Theta}{\partial d_{i}}=0 \Rightarrow d_{i}=\nu_{i}^{\mathrm{t}}\left(P_{i}+T\right)
$$

Note that the above equation can be interpreted as: the optimal depth $d_{i}$ is obtained by orthogonally projecting the translated point onto the projection ray. Similarly applying partial differentiation to $\Theta$ with respect to $T$ and setting it to zero, we have

$$
\frac{\partial \Theta}{\partial T}=0 \Rightarrow T=\frac{1}{N} \sum_{i=1}^{N}\left(d_{i} \nu_{i}-P_{i}\right) .
$$

The above equation can be interpreted as: the optimal translation $T$ is equal to the average distance between the orignal point set and the resulting point set. By substituting eq (16) to eq. (17), we get

$$
\begin{aligned}
T & =\frac{1}{N} \sum_{i=1}^{N}\left(\nu_{i}^{\mathrm{t}}\left(P_{i}+T\right) \nu_{i}-P_{i}\right) \\
& =\frac{1}{N} \sum_{i=1}^{N}\left(\nu_{i}^{\mathrm{t}} P_{i} \nu_{i}-P_{i}\right)+\frac{1}{N} \sum_{i=1}^{N} \nu_{i}^{\mathrm{t}} T \nu_{i} \\
& =\frac{1}{N} \sum_{i=1}^{N}\left[\left(\nu_{i} \nu_{i}^{\mathrm{t}}\right) P_{i}-P_{i}\right]+\frac{1}{N} \sum_{i=1}^{N}\left[\left(\nu_{i} \nu_{i}^{\mathrm{t}}\right) T\right]
\end{aligned}
$$

This implies that

$$
\left(N \cdot I-\sum_{i=1}^{N} \nu_{i} \nu_{i}^{\mathrm{t}}\right) T=\sum_{i=1}^{N}\left(\nu_{i} \nu_{i}^{\mathrm{t}}-I\right) P_{i} .
$$

Writing $A_{i}$ as $I-\nu_{i} \nu_{i}^{\mathrm{t}}$, we have

$$
\begin{aligned}
\left(\sum_{i=1}^{N} A_{i}\right) T & =-\sum_{i=1}^{N} A_{i} P_{i} \\
T & =-\left(\sum_{i=1}^{N} A_{i}\right)^{-1}\left(\sum_{i=1}^{N} A_{i} P_{i}\right) .
\end{aligned}
$$

\section{References}

[1] K. S. Arun, T. S. Huang, and S. D. Blostein. Least-square fitting of two 3-D point sets. IEEE Trans. Pattern Anal. Machine Intell., 9(5):698-700, September 1987. 
[2] A. Azarbayejani and A. Pentland. Recursive estimation of motion, structure, and focal length. IEEE Trans. Pattern Anal. Machine Intell., 17(6):562-575, June 1995.

[3] S. Basu, Irfan Essa, and A. Pentland. Motion regularization for model-based head tracking. TR362, MIT Technical Report, 1996.

[4] T. J. Broida. Recursive 3-D motion estimation from a monocular image sequence. IEEE Trans. Aerospace Electronic Systems, 26(4):639-655, July 1990.

[5] T. J. Broida and R. Chellappa. Estimation of object motion parameters from noisy images. IEEE Trans. Pattern Anal. Machine Intell., 8(1):90-99, January 1986.

[6] D. F. Dementhon and L. S. Davis. Model-based object pose in 25 lines of code. Intl. Journal of Comput. Vision, 15:123-141, 1995.

[7] M. Fischler and Bolles. Random sample consensus: A paradigm for model fitting with applications to image analysis and automated cartography. Commun. ACM, 24(6):381-395, June 1981.

[8] A. Gee and R. Cipolla. Determining the the gaze of faces in images. Image and Vision Computing, 12(10):639-647, December 1994.

[9] D. B. Gennery. Visual tracking of known three-dimensional objects. Intl. Journal of Comput. Vision, 8:243-270, 1992.

[10] D. Goryn and S. Hein. On the estimation of rigid body rotation from noisy data. IEEE Trans. Pattern Anal. Machine Intell., 17(12):1219-1220, December 1995.

[11] B. K. P. Horn. Robot Vision. MIT/McGraw-Hill, New York, 1986.

[12] T. S. Huang and A. N. Netravali. Motion and structure from feature correspondence: A review. Proceedings of the IEEE, 82:252-268, February 1994.

[13] H. Li, P. Roivainen, and R. Forchheimer. 3-D motion estimation in model-based facial image coding. IEEE Trans. Pattern Anal. Machine Intell., 15(6):545-555, June 1993.

[14] D. G. Lowe. Fitting parameterized three dimensional models to images. IEEE Trans. Pattern Anal. Machine Intell., 13(5):441-450, May 1991. 
[15] D. G. Lowe. Robust model-based motion tracking through the integration of search and estimation. Intl. Journal of Comput. Vision, 8:113-122, 1992.

[16] D. P. McReynolds and D. G. Lowe. Rigidity checking of 3D point correspondences under perspective projection. IEEE Trans. Pattern Anal. Machine Intell., 18(12):1174-1185, December 1996.

[17] R. Szeliski and Sing Bing Kang. Recovering 3D shape and motion from image streams using non-linear least squares. Cambridge Research Laboratory Technical Report, March 1993.

[18] R. Y. Tsai. A versatile camera calibration technique for high accuracy 3D machine vision metrology using off-the-shelf TV cameras and lenses. IEEE Trans. Robotics and Automation, RA3(4):323-344, August 1987.

[19] Shinji Umeyama. Least-square estimation of transformation parameters between two point pattern. IEEE Trans. Pattern Anal. Machine Intell., 13(4):376-380, April 1991.

[20] K. Waters. A muscle model for animating three-dimensional facial expression. Comput. Graphics, 21(4):17-24, 1987.

[21] J. Weng, N. Ahuja, and T. S. Huang. Optimal motion and structure estimation. IEEE Trans. Pattern Anal. Machine Intell., 15(9):864-884, September 1993.

[22] P. Wunsch and G. Hirzinger. Registration of CAD-models to images by iterative inverse perspective matching. Proc. ICPR 96, pages 78-83, November 1996.

[23] Z. Zhang and O. Faugeras. 3D Dynamic Scene Analysis. Springer-Verlag, 1992. 


\section{List of Figures}

1 Relationship between original point and projected point . . . . . . . . . . 22

2 CPU time per iteration for Extended Kalman Filter, Gauss-Newton and PRA . . . 23

3 Recovered angles vs. input angles . . . . . . . . . . . . . . . . . 24

4 Recovered values for increasing translation . . . . . . . . . . . . . 25

5 Plot of error dependency on the number of points . . . . . . . . . 26

6 Plot of error in estimation vs. measurement noise . . . . . . . . . . . 27

7 Sample run of the head tracking application. a) initialization, . . . . . . . . . 28

7 b) Rotation of the head and reconstructed pose, . . . . . . . . . . . 28 


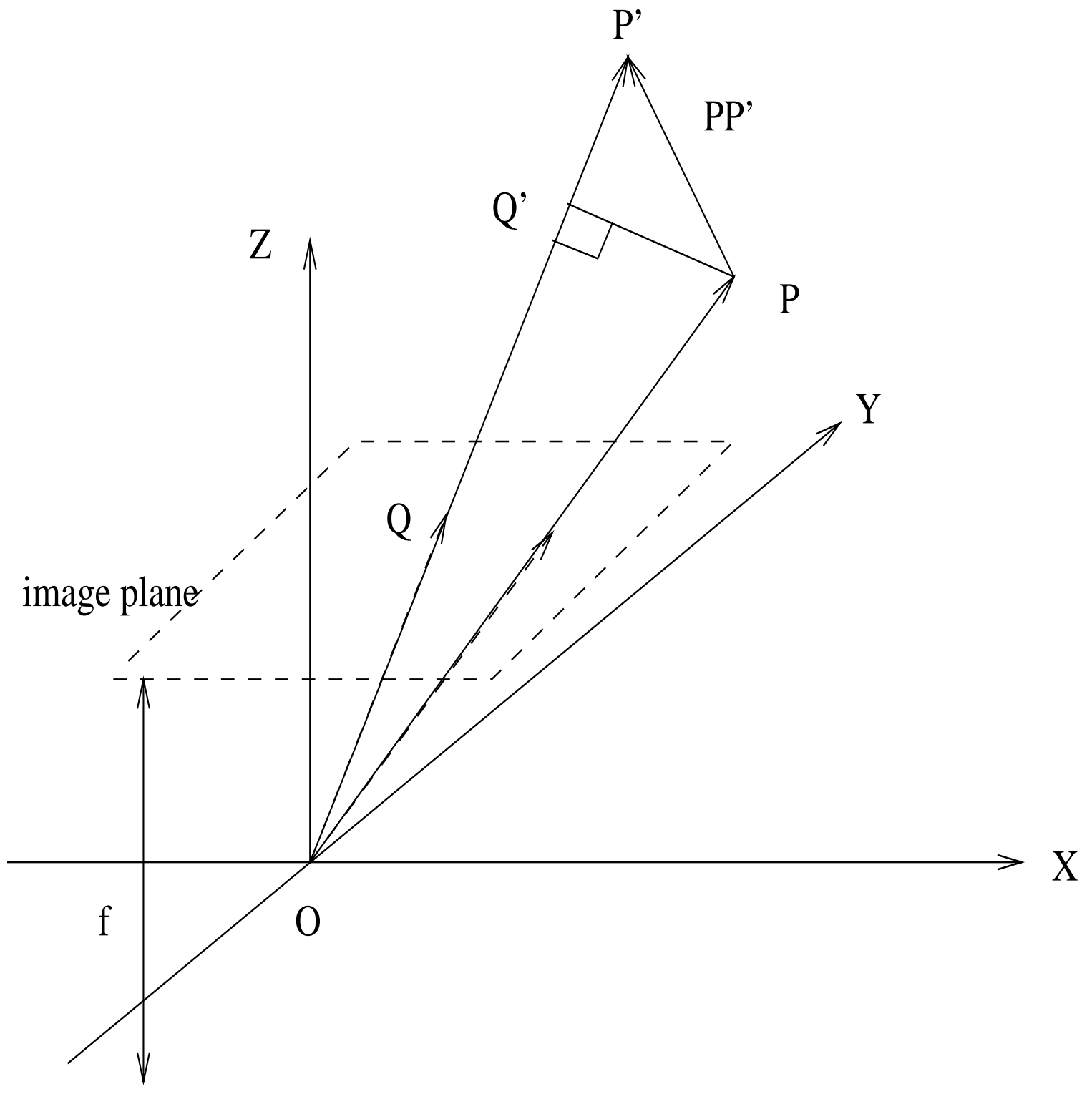

Figure 1: Image and Vision Computing $\quad$ S.H. Or,W.S. Luk, K.H. Wong, I. King 
Fig. 2. CPU time per iteration for Extended Kalman Filter, Gauss-

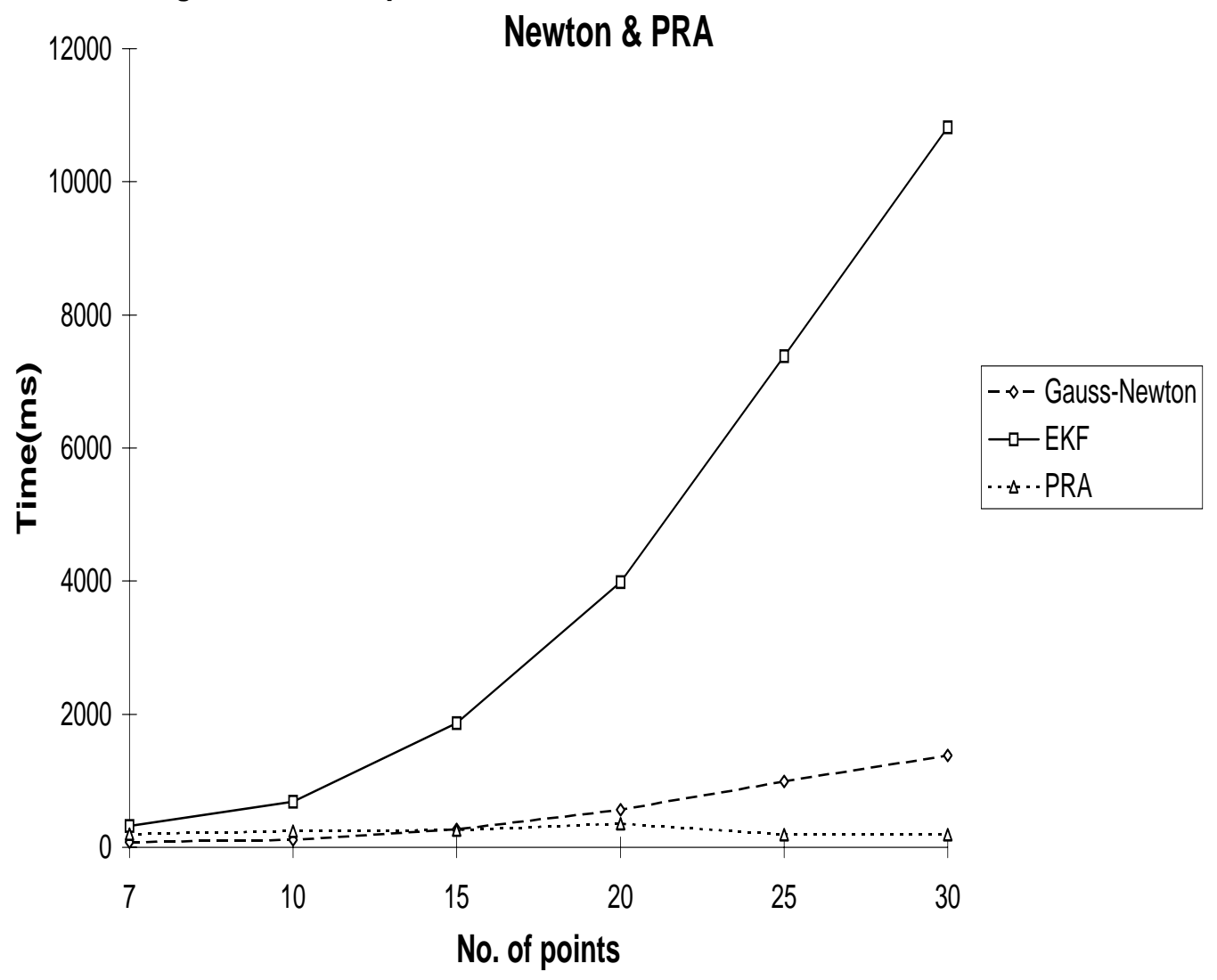

Figure 2: Image and Vision Computing $\quad$ S.H. Or,W.S. Luk, K.H. Wong, I. King 


\section{Fig.3 Recovered Angles vs. Input Angles}

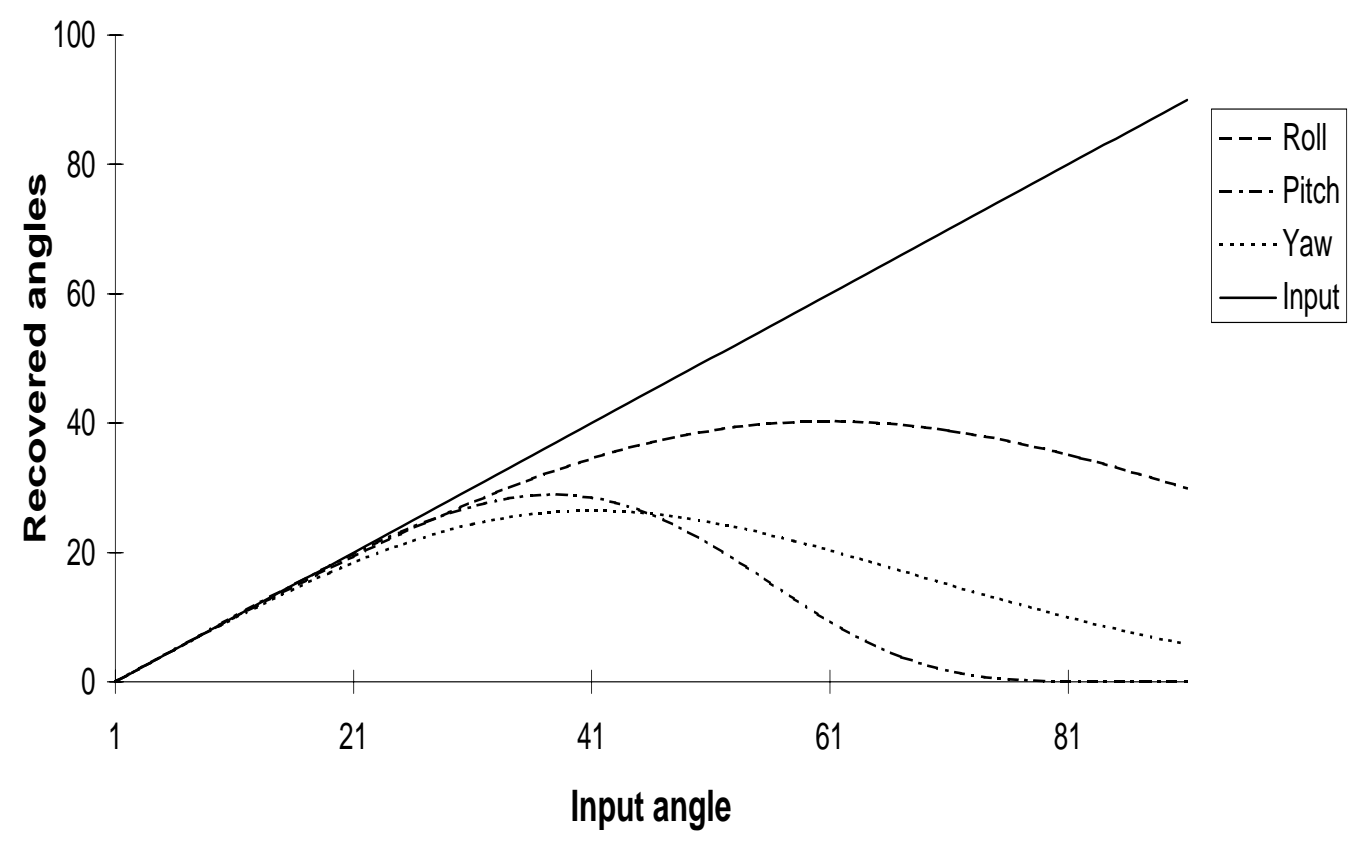

Figure 3: Image and Vision Computing $\quad$ S.H. Or,W.S. Luk, K.H. Wong, I. King 
Fig. 4 Recovered values for increasing translation

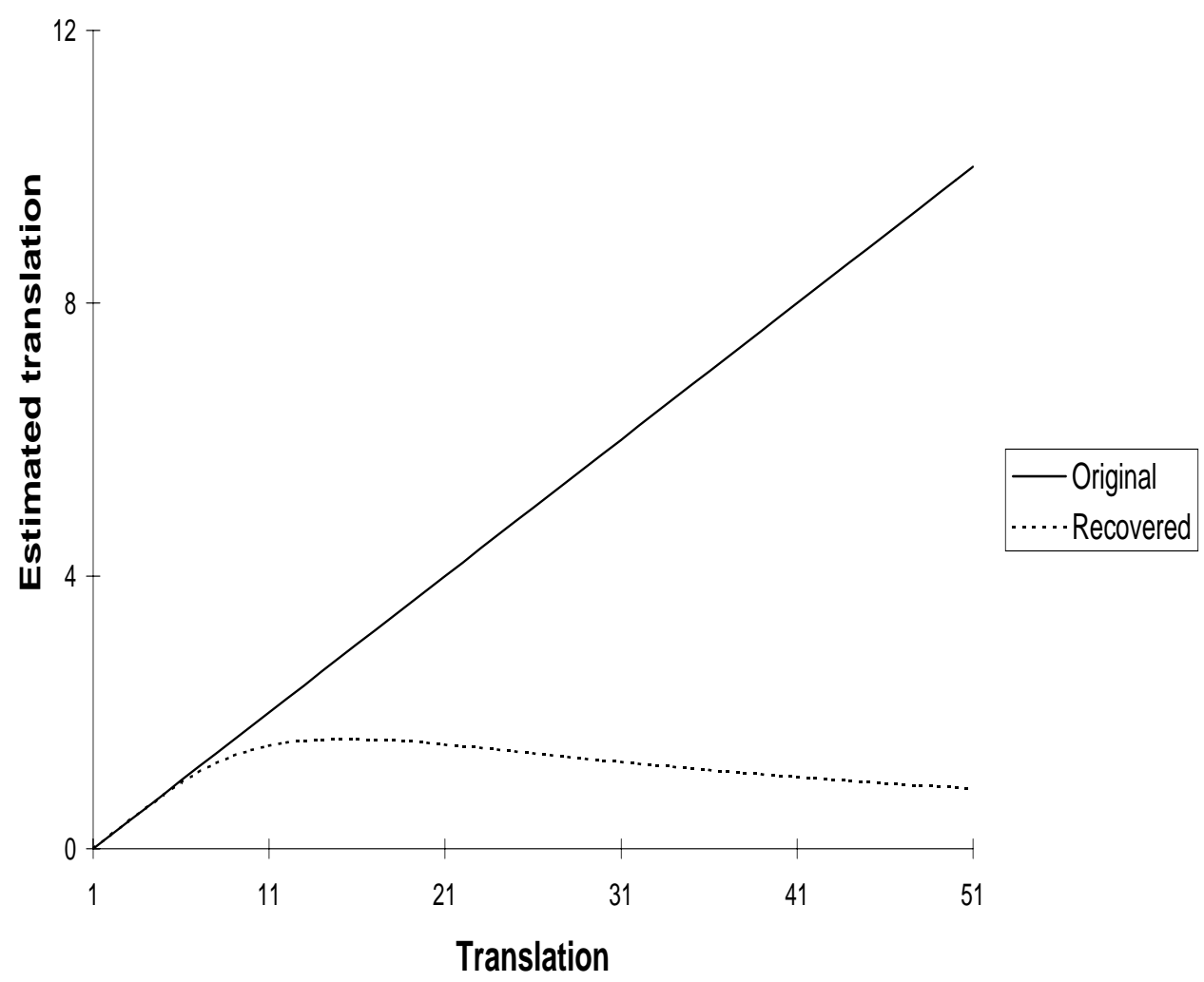

Figure 4: Image and Vision Computing S.H. Or,W.S. Luk, K.H. Wong, I. King 
Fig. 5 Plot of error dependency on the number of points

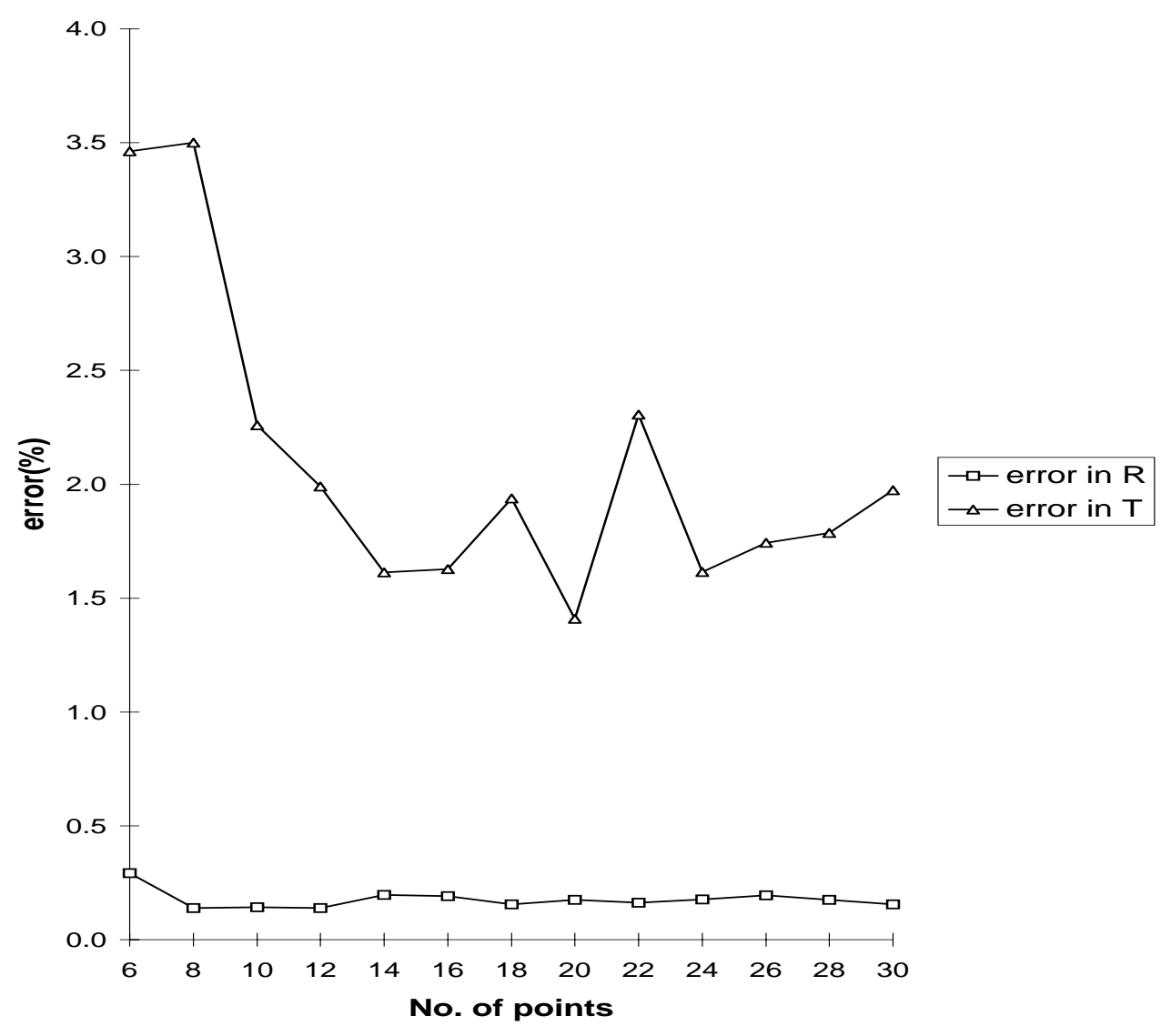

Figure 5: Image and Vision Computing $\quad$ S.H. Or,W.S. Luk, K.H. Wong, I. King 
Fig. 6 Plot of error in estimation vs. measurement noise

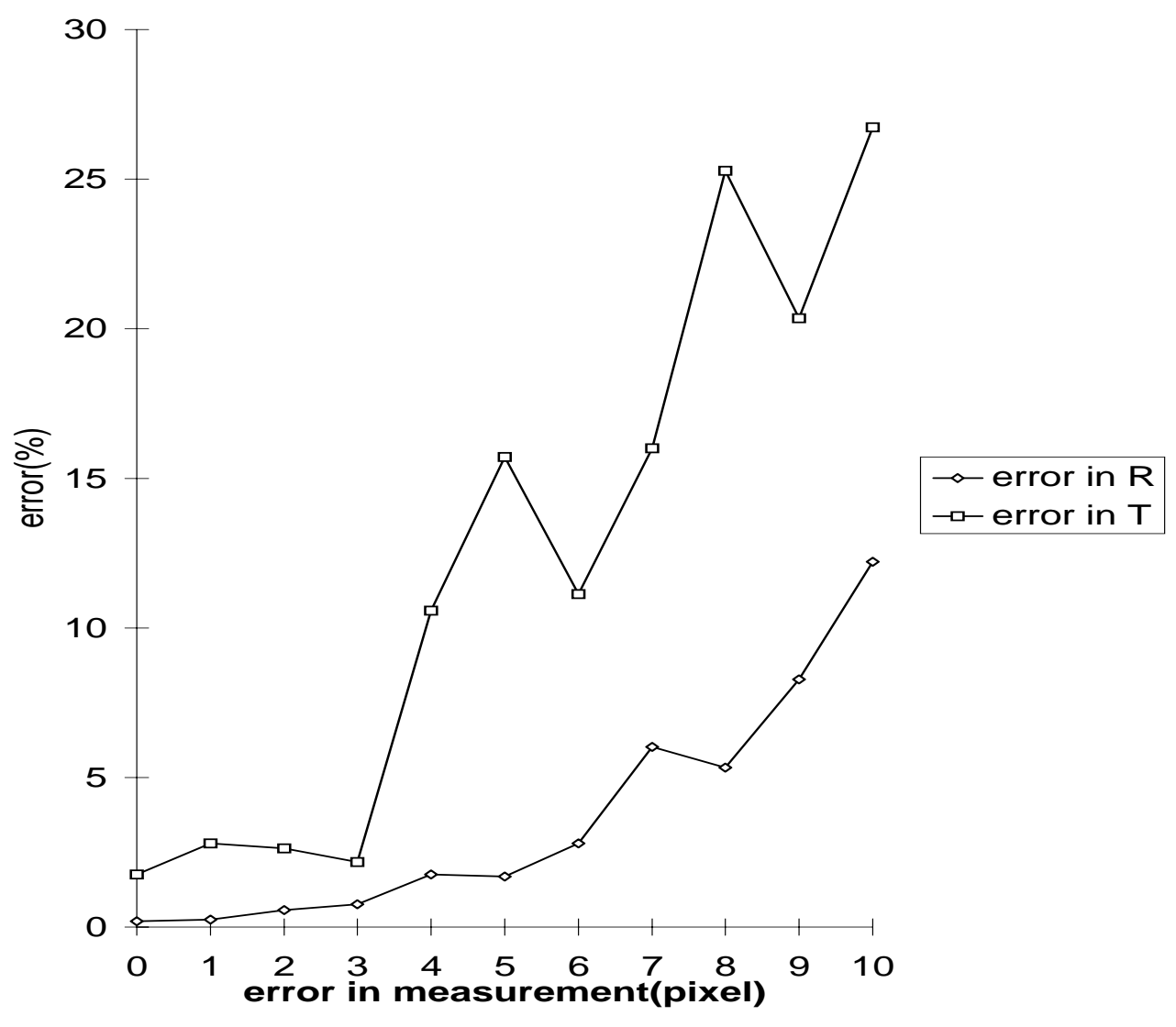

Figure 6: Image and Vision Computing $\quad$ S.H. Or,W.S. Luk, K.H. Wong, I. King 


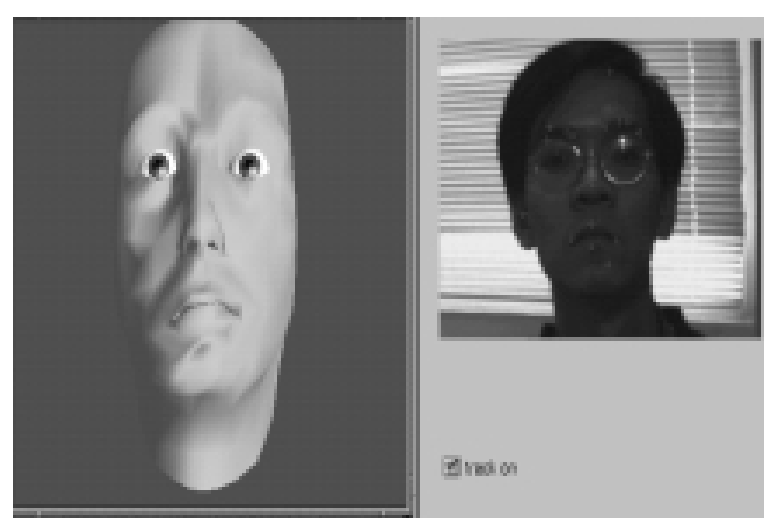

Figure 7: a)

Image and Vision Computing

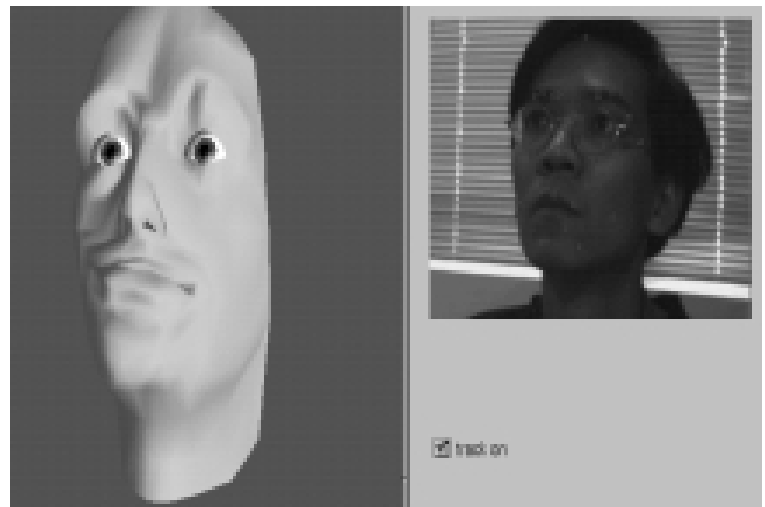

Figure 7: b)

S.H. Or,W.S. Luk, K.H. Wong, I. King 